\title{
TECNOLOGIAS DIGITAIS E DE GEOPROCESSAMENTO APLICADAS AO MONITORAMENTO DA DOENÇA DE CORONOVÍRUS 2019 (COVID-19)
}

\section{DIGITAL AND GEOPROCESSING TECHNOLOGIES APPLIED TO MONITORING CORONOVIRUS DISEASE 2019 (COVID-19)}

\author{
Claudio José dos Santos Júnior \\ Mestrando. Programa de Pós-Graduação em Ensino na Saúde e Tecnologia \\ Universidade Estadual de Ciências da Saúde de Alagoas (UNCISAL), Maceió (AL), Brasil \\ claudio.junior@uncisal.edu.br \\ Paulo José Medeiros de Souza Costa \\ Doutor. Programa de Pós-Graduação em Ensino na Saúde e Tecnologia \\ Universidade Estadual de Ciências da Saúde de Alagoas (UNCISAL), Maceió (AL), Brasil \\ paulojmsc@yahoo.com.br
}

John Victor dos Santos Silva

Mestrando. Programa de Pós-Graduação em Enfermagem Universidade de São Paulo (USP), Ribeirão Preto (SP), Brasil johnvictor@usp.br

Mestre em Ciências da Saúde. Una Kelly Pinheiro Souza arynnakelly@gmail.com

Jackson Pinto Silva

Mestre em Análise de Sistemas Ambientais. Geógrafo. Instituto Federal de Alagoas - Campus Maceió (IFAL) jacksonpinto@bol.com.br

Thiago José Matos Rocha

Doutor em Inovação Terapêutica. Mestre em Medicina Tropical Universidade Estadual de Ciências da Saúde de Alagoas (UNCISAL)

thy rocha@hotmail.com

\begin{abstract}
RESUMO
Objetivo: Realizar levantamento das principais tecnologias de mapeamento, monitoramento e disseminação de informações geográfico-epidemiológicas sobre a COVID19. Métodos: Trata-se de estudo exploratório que utilizou a bibliometria e a análise de conteúdo como principais ferramentas metodológicas. Resultados: Foram identificadas 31 tecnologias digitais de mapeamento, monitoramento e disseminação de informações geográfico-epidemiológicas sobre a COVID-19 disponíveis na web. Do total de recursos, 13 tecnologias tinham abrangência internacional e 18 possuiam caráter locorregional e/ou nacional. Predominaram tecnologias do tipo "mapas" e "gráficos", nesta ordem. Conclusão: Fora produzido um levantamento que reúne as principais geotecnologias digitais sobre a COVID-19. Esse material poderá servir de subsídio as ações de vigilância em saúde e ser utilizado como guia para elucidar aspectos relacionados ao panorama de dúvidas que predomina quanto ao comportamento epidemiológico do novo coronavírus.
\end{abstract}

Palavras-chave: Novo coronavírus. SARS-CoV-2. Geoprocessamento. Tecnologias digitais. Epidemiologia.

\section{ABSTRACT}

Objective: To map the main technologies for mapping, monitoring and disseminating geographic-epidemiological information about COVID-19. Methods: This is an exploratory study that used bibliometrics and content analysis as the main methodological tools.

Recebido em: 20/04/2020

Aceito para publicação em: 21/05/2020. 
Tecnologias digitais e de geoprocessamento aplicadas ao monitoramento da doença de Coronovírus 2019 (Covid-19)
Claudio José dos Santos Júnior Paulo José Medeiros de Souza Costa John Victor dos Santos Silva Aryanna Kelly Pinheiro Souza Jackson Pinto Silva

Thiago José Matos Rocha

Results: 31 digital technologies for mapping, monitoring and disseminating geographicepidemiological information about COVID-19 available on the web were identified. Of the total resources, 13 technologies had an international scope and 18 had a locoregional and / or national character. Technologies such as "maps" and "graphics" predominated, in that order. Conclusion: A survey had been produced that gathers the main digital geotechnologies about COVID-19. This material can serve as a basis for epidemiological surveillance actions and can be used as a guide to elucidate aspects of the prevailing doubts regarding the behavior of the new coronavirus.

Keywords: New coronavirus. SARS-CoV-2. Geoprocessing. Digital technologies. Epidemiology.

\section{INTRODUÇÃO}

O uso das tecnologias digitais está cada vez mais presente nos serviços de saúde, seja no atendimento ao paciente, seja nos processos de gestão dos sistemas e serviços de saúde. Entre as tecnologias digitais de processamento e análise de dados, destaca-se o geoprocessamento - uma ferramenta de gestão que permite a análise da distribuição espacial de determinado agravo de saúde em um território, viabilizando, assim, ações direcionadas e executadas com maior efetividade e assertividade (BONIFÁCIO; LOPES, 2019).

O geoprocessamento, ao mesmo tempo em que constitui-se num conjunto de tecnologias de processamento de dados, é considerado uma área do conhecimento que engloba os Sistemas de Informação Geográfica (SIG), o sensoriamento remoto e as técnicas de análise espacial, possuindo interfaces com a Cartografia, a Geografia, a Estatística, a Matemática, entre outras ciências (CHIARAVALLOTI-NETO, 2016).

As geotecnologias, dessa forma, baseiam-se num conjunto de informações que podem ser expressas em formato de mapas e diagramas digitais (GOLDSTEIN et al., 2013). Através das técnicas e aplicações geoespaciais é possível identificar, localizar, monitorar e acompanhar populações e agravos (RIBEIRO et al., 2014).

A criação de mapas, cartogramas e afins, a partir das técnicas e dos sistemas de informação geográfica, configura-se como instrumento útil para o apoio e a estruturação das ações de vigilância epidemiológica, aqui entendida como o conjunto de ações que proporcionam o conhecimento, a detecção ou prevenção de qualquer mudança nos fatores determinantes e condicionantes de saúde individual ou coletiva, com a finalidade de recomendar e adotar as medidas de prevenção e controle das doenças ou agravos (ARREAZA; MORAES, 2010).

A importância do geoprocessamento e dos métodos de análise de dados espaciais como ferramenta de investigação epidemiológica situa-se na suas múltiplas possibilidades de fornecer informações aos gestores em saúde para tomada de decisão sobre a execução de ações de controle de doenças e agravos, especificamente no que tange à coleta, processamento e interpretação de dados, bem como no que tange à divulgação de informações, à investigação epidemiológica de casos e surtos, à análise dos resultados obtidos e ao fornecimento de recomendações e medidas de controle (BRASIL, 1990; BONIFÁCIO; LOPES, 2019).

As técnicas de geoprocessamento, nesse contexto, configuram-se como importantes ferramentas de suporte as ações de pr, vigilância e controle de doenças em um território (RIBEIRO et al., 2018). Constituem-se, ademais, em instrumentos capazes de contribuir para o planejamento e avaliação das ações de saúde, de forma a mapear, monitorar e a disseminar as informações em saúde por meio de recursos que proporcionarão melhoria da qualidade da informação e, por consequência, atuação direcionada e mais efetiva (RIBEIRO et al., 2014).

O Brasil e o mundo vivem uma emergência em saúde pública desde o início da atual pandemia de coronavírus (SARS-CoV-2), causador da COVID-19. Tal situação tem gerado grandes preocupações em várias nações do mundo, além de impactos socioeconômicos de importantes magnitudes. De acordo com a Organização Mundial da Saúde (OMS), em 17 de maio de 2020, os casos confirmados 
Tecnologias digitais e de geoprocessamento aplicadas ao monitoramento da doença de Coronovírus 2019 (Covid-19)
Claudio José dos Santos Júnior Paulo José Medeiros de Souza Costa John Victor dos Santos Silva Aryanna Kelly Pinheiro Souza Jackson Pinto Silva

Thiago José Matos Rocha

da COVID-19 já haviam ultrapassado 4,7 milhões em todo o mundo e somavam em torno de 314 mil óbitos (WHO, 2020).

Nesse contexto, tendo em vista a contribuição das técnicas geoespaciais como instrumento de gestão em saúde, a utilização do geoprocessamento na vigilância e controle da COVID-19 mostra-se como recurso em potencial para o planejamento e avaliação de ações nessa área, uma vez que com tais recursos é possível mapear, monitorar e disseminar dados acerca da COVID-19 em um território, podendo-se, assim, traçar cenários e ações direcionadas.

\section{OBJETIVO}

Este estudo objetivou levantar as tecnologias digitais de mapeamento, monitoramento e disseminação de informações geográfico-epidemiológicas sobre a COVID-19.

\section{MÉTODOS}

Trata-se de estudo exploratório que utilizou a bibliometria e a análise de conteúdo virtual como principais ferramentas metodológicas.

A utilização da análise bibliométrica em pesquisas científicas se pauta na investigação do comportamento do conhecimento e da literatura como parte dos processos de comunicação. Embora tal técnica tenha sua maior aplicação nos campos da Ciência da Informação, é possível aplicá-la em várias áreas do conhecimento a fim de explorar o impacto da produção de um determinado campo de conhecimento, além da produção e da produtividade de um conjunto de investigadores e de temas, por meio da construção de indicadores bibliométricos (MARCELO; HAYASHI, 2013).

Neste trabalho, foi realizada a exploração dos conteúdos virtuais referentes ao tema COVID-19 disponíveis na web, através de análise bibliométrica, para levantamento de recursos de mapeamento, monitoramento e disseminação de informação geográfico-epidemiológica.

Foram realizadas buscas nas plataformas Google (www.google.com.br), Bing (br.bing.com), Yahoo (br.yahoo.com), Ask (ask.com) e AOL (aol.com). Os referidos sites de busca foram selecionados por compreenderem as principais plataformas de busca de conteúdo existente na internet, sendo acessados por bilhões de pessoas no Brasil e no mundo.

A seleção do material (coleta de dados) ocorreu em um único momento, no dia 18 de abril de 2020, das $08 \mathrm{~h}$ às $19 \mathrm{~h}$. Para tanto, definiram-se as seguintes palavras-chave "coronavírus" (coronavirus), "COVID-19" (COVID-19), "tecnologia digital" (digital technology) e "geoprocessamento" (geoprocessing) utilizados no português e em inglês, de forma associada por meio de diferentes combinações através do operador boleano AND, e com o uso de aspas como estratégia de restringir a pesquisa a materiais que tivessem os descritores de interesse em seu conteúdo.

Todos os links presentes nas 10 (dez) primeiras páginas de resultados de cada um dos buscadores eletrônicos foram considerados e analisados individualmente. Tal parâmetro foi baseado em outros trabalhos que verificaram que os usuários não costumam visualizar um número de páginas superior ao descrito (MURUGIAH et al., 2011; CARVALHO et al., 2017).

A respeito do critério de inclusão, consideraram-se páginas da web que apresentavam conteúdo relacionado ao tema COVID-19, que veiculassem informações epidemiológicas sobre o tema central no âmbito nacional e/ou internacional, e que fossem compatíveis com alguma das modalidades de tecnologia digital de informação, comunicação ou geoprocessamento em saúde existentes.

Foram excluídos os conteúdos duplicados, que apresentavam baixa qualidade audiovisual e de imagem e aqueles que não apresentavam fonte primária de dados claramente evidenciada no material consultado e/ou na página de vinculação.

A coleta de dados foi realizada aos pares, de forma independente, com auxílio de formulário específico, o qual continha informações relativas às seguintes características gerais da tecnologia: tipo de tecnologia digital; título; funcionalidades; data da postagem; link de acesso; desenvolvedor; e abrangência territorial.

DOl:http://dx.doi.org/10.14393/Hygeia0053912 $\quad$ Hygeia $\quad$ Edição Especial: Covid-19, Jun./2020 p.1 - 10, pág. 3


Tecnologias digitais e de geoprocessamento aplicadas ao monitoramento da doença de Coronovírus 2019 (Covid-19)
Claudio José dos Santos Júnior Paulo José Medeiros de Souza Costa John Victor dos Santos Silva Aryanna Kelly Pinheiro Souza Jackson Pinto Silva

Thiago José Matos Rocha

Como forma de efetuar a classificação das tecnologias, utilizaram-se as informações disponíveis no próprio site do desenvolvedor.

A busca inicial com a inserção dos descritores mencionados resultou em 95 páginas da web, após exclusão dos conteúdos repetidos. Após pré-seleção a partir de visitação ao endereço, consulta ao título, aplicação dos critérios estabelecidos, visualização do conteúdo e do consenso entre os pesquisadores sobre os conteúdos audiovisuais, obteve-se uma amostra igual a 31 tecnologias digitais de informação e comunicação sobre o tema em estudo.

Os dados foram agrupados em banco de dados por meio do programa Microsoft Excel 2017 e foram analisados de forma descritiva, tendo sido apresentados através de quadros.

Por tratar-se de um estudo realizado com dados secundários e de domínio público, disponibilizados na internet, não houve a necessidade de apreciação por Comitê de Ética em Pesquisa, sendo dispensada, também, a utilização do Termo de Consentimento Livre e Esclarecido.

\section{RESULTADOS}

Foram identificadas 31 tecnologias digitais de mapeamento, monitoramento e disseminação de informações geográfico-epidemiológicas sobre a COVID-19 disponíveis na web. Do total de recursos, 13 tecnologias tinham abrangência internacional (Quadro 1) e 18 possuíam caráter locorregional e/ou nacional e visavam a abordagem da situação epidemiológica da COVID-19 no território do Brasil (Quadro 2). Tecnologias do tipo "mapas" e "gráficos" interativos apresentaram maior ocorrência, nesta ordem.

O conteúdo das tecnologias digitais foi bastante diverso. A maior parte dos recursos trata da apresentação dos casos por localidade (investigados, infectados, confirmados, óbitos, entre outras variações), da abordagem de taxas e indicadores de morbimortalidade (incidência, prevalência, letalidade) e da apresentação de projeções de variáveis epidemiológicas em distintos cenários.

Há, do mesmo modo, um conjunto de tecnologias que se preocupa em abordar o perfil de indivíduos, dados de emprego e renda das regiões, informações sobre a rede hospitalar e demanda de leitos, além de recursos que apresentam informações como óbitos por grupo de risco, por perfil etário e por sexo do indivíduo acometido.

Nos quadros 1 e 2 é possível identificarmos as principais características de cada uma das tecnologias, além de sua abrangência, página de acesso, funcionalidades e instituição desenvolvedora.

Quadro 1 - Distribuição das Tecnologias digitais sobre o tema COVID-19 com abrangência internacional $(\mathrm{N}=13)$.

\begin{tabular}{|l|l|l|l|}
\hline \multicolumn{1}{|c|}{ Identificação } & Desenvolvedor & Abrangência & Descrição / Funcionalidade \\
\hline $\begin{array}{l}\text { Global cases COVID-19 } \\
\text { (coronavirus.jhu.edu/map) }\end{array}$ & $\begin{array}{l}\text { Universidade Johns } \\
\text { Hopkins }\end{array}$ & $\begin{array}{l}\text { Mundial (Países) } \\
\text { Casos de coronavirus } \\
\text { (worldometers.info/coronavirus) }\end{array}$ & $\begin{array}{l}\text { Mapa interativo representa total de casos confirmados } \\
\text { e óbitos por país, estados e capitais. }\end{array}$ \\
\hline & & $\begin{array}{l}\text { Mundial (Países) } \\
\text { Gráficos interativos apresentam progressão dos casos } \\
\text { por país; progressão das mortes por país; linha do } \\
\text { tempo dos casos; total de casos novos; casos diários, } \\
\text { casos ativos, recuperados e em investigação; casos } \\
\text { graves e críticos; total de mortes e mortes diárias. } \\
\text { Apresenta, também, projeções linear e logarítmica } \\
\text { para os dados. }\end{array}$ \\
\hline
\end{tabular}


Tecnologias digitais e de geoprocessamento aplicadas ao monitoramento da doença de Coronovírus 2019 (Covid-19)
Claudio José dos Santos Júnior Paulo José Medeiros de Souza Costa John Victor dos Santos Silva Aryanna Kelly Pinheiro Souza Jackson Pinto Silva

Thiago José Matos Rocha

\begin{tabular}{|c|c|c|c|}
\hline $\begin{array}{l}\text { Covid Trends } \\
\text { (www.aatishb.com/covidtrends) }\end{array}$ & Minute Physics & Mundial (Países) & $\begin{array}{l}\text { Gráfico interativo mostra os casos novos e } \\
\text { confirmados de COVID-19 na semana anterior versus } \\
\text { o total de casos confirmados até o momento. Fornece } \\
\text { informações, também, sobre os óbitos e expressa o } \\
\text { crescimento exponencial do número de casos, além de } \\
\text { permitir fazer projeções logarítmicas. }\end{array}$ \\
\hline $\begin{array}{l}\text { Covid Visualizer } \\
\text { (www.covidvisualizer.com) }\end{array}$ & $\begin{array}{l}\text { Carnegie Mellon } \\
\text { University }\end{array}$ & Mundial (Países) & $\begin{array}{l}\text { Mapa interativo apresenta informações sobre número } \\
\text { de casos, mortes e recuperações por COVID-19 por } \\
\text { país do mundo. O objetivo da ferramenta é fornecer de } \\
\text { maneira simples e interativa o impacto da COVID-19 } \\
\text { em diferentes localidades do mundo. }\end{array}$ \\
\hline $\begin{array}{l}\text { COVID-19 Global } \\
\text { (covid19info.live) }\end{array}$ & Shortbread & Mundial (Países) & $\begin{array}{l}\text { Gráficos e mapas interativos revelam a quantidade de } \\
\text { casos confirmados, óbitos, casos } \\
\text { recuperados e infectados (cumulativamente e } \\
\text { diariamente) em diferentes países do mundo. }\end{array}$ \\
\hline $\begin{array}{l}\text { Mapa do coronavírus } \\
\text { (google.com/covid19-map) }\end{array}$ & Google & \begin{tabular}{|l} 
Mundial \\
(Continentes e \\
Países)
\end{tabular} & $\begin{array}{l}\text { Mapa interativo apresenta casos por milhão de } \\
\text { pessoas, número absoluto de casos confirmados, } \\
\text { recuperados e mortes. Os casos incluem pessoas que } \\
\text { tiveram testes com resultado positivo. Utiliza dados } \\
\text { dos relatórios sobre a situação diária disponíveis no } \\
\text { site da Organização Mundial da Saúde. }\end{array}$ \\
\hline $\begin{array}{l}\text { Nextstrain - rastreamento em temp } \\
\text { real da evolução de patógenos } \\
\text { (www.nextstrain.org/ncov/global) }\end{array}$ & Bioinformatics & Mundial (Países) & $\begin{array}{l}\text { Gráfico interativo em formato de filogenia mostra } \\
\text { relações evolutivas do vírus SARS-CoV-2. Mostra um } \\
\text { surgimento inicial em Wuhan, China, no final de } 2019 \text {, } \\
\text { seguido por uma transmissão sustentada de humano } \\
\text { para humano, levando a infecções amostradas e, com } \\
\text { base em estimativas de datas de transmissão } \\
\text { específicas, infere padrões de transmissão específicos } \\
\text { e reconstrói a distribuição geográfica da doença. }\end{array}$ \\
\hline $\begin{array}{l}\text { Rastreador do COVID-19 } \\
\text { (bing.com/covid) }\end{array}$ & Microsoft & Mundial (Países) & $\begin{array}{l}\text { Mapa interativo mostra a quantidade de casos ativos, } \\
\text { recuperados e fatais da COVID-19 em diferentes } \\
\text { países ao redor do globo. }\end{array}$ \\
\hline $\begin{array}{l}\text { Coronavirus (COVID-19) } \\
\text { (who.sprinklr.com/) }\end{array}$ & WHO & Mundial (Países) & $\begin{array}{l}\text { Mapa e gráfico interativos apresentam dados sobre } \\
\text { casos confirmados (novos e total) variação diária e } \\
\text { mortes por COVID-19 nos países e continentes. } \\
\text { Estratifica as curvas de registro por nação e permite } \\
\text { avaliar a situação epidemiológica por dia do mês. }\end{array}$ \\
\hline $\begin{array}{l}\text { COVID-19 Community Mobility } \\
\text { (google.com/covid19/mobility) }\end{array}$ & Google & Mundial (Países) & $\begin{array}{l}\text { Site apresenta relatórios de mobilidade da comunidade } \\
\text { que visam fornecer informações sobre o que mudou } \\
\text { em resposta às políticas destinadas a combater à } \\
\text { COVID-19. Os relatórios traçam tendências de } \\
\text { movimento ao longo do tempo por região geográfica, } \\
\text { em diferentes categorias de lugares, como varejo e } \\
\text { recreação, compras e farmácias, parques, estações de } \\
\text { trânsito, locais de trabalho e residenciais. }\end{array}$ \\
\hline \begin{tabular}{|l} 
Data on COVID-19 testing \\
(ourworldindata.org/covid-testing)
\end{tabular} & Our World in Data & Mundial (Países) & $\begin{array}{l}\text { Mapa e gráfico interativos apresentam dados de } \\
\text { testagem de COVID-19 em vários países ao redor do } \\
\text { mundo. O objetivo é fornecer entendimento global da } \\
\text { situação de cada país e das limitações importantes } \\
\text { que as nações podem apresentar. }\end{array}$ \\
\hline $\begin{array}{l}\text { Mapa do coronavírus } \\
\text { (bit.ly/2VdBQVh) }\end{array}$ & Channel News Asia & Mundial (Países) & $\begin{array}{l}\text { Mapa interativo ilustra a quantidade de casos } \\
\text { confirmados, óbitos e casos recuperados em diferentes } \\
\text { países do mundo. }\end{array}$ \\
\hline $\begin{array}{l}\text { Ponto de Situação Atual em } \\
\text { Portugal } \\
\text { (covid19.min-saude.pt/ponto-de- } \\
\text { situacao-atual-em-portugal) }\end{array}$ & DGS Portugal & \begin{tabular}{|l} 
País \\
(Portugal)
\end{tabular} & $\begin{array}{l}\text { Mapa interativo ilustra casos em acompanhamento, } \\
\text { que aguardam resultado laboratorial, em vigilância } \\
\text { pelas autoridades, em internamento, casos internados } \\
\text { e casos internados em unidades de cuidados } \\
\text { intensivos. }\end{array}$ \\
\hline
\end{tabular}

Fonte: Elaborado pelos autores (2020). 
Tecnologias digitais e de geoprocessamento aplicadas ao monitoramento da doença de Coronovírus 2019 (Covid-19)
Claudio José dos Santos Júnior Paulo José Medeiros de Souza Costa John Victor dos Santos Silva Aryanna Kelly Pinheiro Souza Jackson Pinto Silva

Thiago José Matos Rocha

Quadro 2 - Distribuição das Tecnologias digitais sobre o tema "COVID-19" com abrangência nacional $(\mathrm{N}=18)$.

\begin{tabular}{|c|c|c|c|}
\hline Tecnologia & Desenvolvedor & Abrangência & Descrição \\
\hline $\begin{array}{l}\text { Painel Coronavírus Brasil } \\
\text { (covid.saude.gov.br) }\end{array}$ & Ministério da Saúde & $\begin{array}{l}\text { Nacional } \\
\text { (Estados) }\end{array}$ & $\begin{array}{l}\text { Mapas e gráficos interativos representam total de casos } \\
\text { confirmados, óbitos, letalidade, casos por região, casos por } \\
\text { dia, casos por estado, casos por semana epidemiológica e } \\
\text { casos acumulados. }\end{array}$ \\
\hline $\begin{array}{l}\text { Coronavírus } \\
\text { (sigageomarketing.com.br/coronavi } \\
\text { rus) }\end{array}$ & i Siga Geomarketing & $\begin{array}{l}\text { Nacional } \\
\text { (Estados) }\end{array}$ & $\begin{array}{l}\text { Site apresenta o total de casos confirmados e óbitos por } \\
\text { Unidade Federativa do Brasil. }\end{array}$ \\
\hline $\begin{array}{l}\text { Informes COVID-19 } \\
\text { (nexojornal.com.br/grafico) }\end{array}$ & Nexo Jornal & Nacional & $\begin{array}{l}\text { Site apresenta gráficos da distribuição dos respiradores no } \\
\text { Brasil antes da pandemia e das projeções para a COVID- } \\
19 \text { no Brasil em diferentes cenários. }\end{array}$ \\
\hline $\begin{array}{l}\text { Mapa Brasileiro da COVID-19 } \\
\text { (inloco.com.br/pt/) }\end{array}$ & inloco Soluções & $\begin{array}{l}\text { Nacional } \\
\text { (Estados) }\end{array}$ & $\begin{array}{l}\text { Mapa interativo expressa o índice de isolamento social e } \\
\text { ranking dos estados para auxiliar no combate à pandemia. } \\
\text { O mapa mostra o percentual, por estado, da população que } \\
\text { está respeitando a recomendação de isolamento. Através } \\
\text { dessa ferramenta as autoridades podem direcionar } \\
\text { recursos de saúde, segurança e comunicação. }\end{array}$ \\
\hline $\begin{array}{l}\text { Mapa do Coronavírus } \\
\text { (mapadocoronavirus.com) }\end{array}$ & \begin{tabular}{|l|} 
Produção \\
independente
\end{tabular} & $\begin{array}{l}\text { Nacional } \\
\text { (Estados e } \\
\text { Regiões) }\end{array}$ & $\begin{array}{l}\text { Mapa interativo apresenta casos suspeitos, confirmados, } \\
\text { graves, recuperados e mortes por cidade/estado do Brasil. }\end{array}$ \\
\hline $\begin{array}{l}\text { Monitor Covid por estado } \\
\text { (bit.ly/34ol2y3) }\end{array}$ & Estadão & $\begin{array}{l}\text { Nacional } \\
\text { (Estado) }\end{array}$ & $\begin{array}{l}\text { Gráficos ilustra a quantidade absoluta e incidência de } \\
\text { casos por habitantes por estado do Brasil. }\end{array}$ \\
\hline $\begin{array}{l}\text { Número de casos confirmados de } \\
\text { COVID-19 no Brasil } \\
\text { (labs.wesleycota.com/sarscov2/br) }\end{array}$ & $\begin{array}{l}\text { Universidade } \\
\text { Federal de Viçosa }\end{array}$ & $\begin{array}{l}\text { Nacional } \\
\text { (Municípios) }\end{array}$ & $\begin{array}{l}\text { Mapa interativo apresenta os dados de casos novos e } \\
\text { óbitos por COVID-19 no Brasil. As fontes de informação } \\
\text { são a plataforma oficial do Ministério da Saúde e das } \\
\text { secretarias de saúde de cada estado. }\end{array}$ \\
\hline $\begin{array}{l}\text { Observatório COVID-19 BR } \\
\text { (covid19br.github.io) }\end{array}$ & IB-USP e outros & $\begin{array}{l}\text { Nacional } \\
\text { (Estados e } \\
\text { Regiões) }\end{array}$ & $\begin{array}{l}\text { Site apresenta gráficos, mapas e projetações da } \\
\text { quantidade de casos notificados no Brasil. Apresenta um } \\
\text { modelo virtual de propagação do vírus e estimativa do } \\
\text { tempo de duplicação por localidade. }\end{array}$ \\
\hline $\begin{array}{l}\text { Painel Rio COVID-19 } \\
\text { (bit.ly/2xYPgN1) }\end{array}$ & SMS-RJ & $\begin{array}{l}\text { Local (Município } \\
\text { do Rio de } \\
\text { Janeiro-RJ) }\end{array}$ & $\begin{array}{l}\text { Mapa e gráfico interativos representam acumulado de } \\
\text { óbitos, acumulado de casos confirmados, evolução diária } \\
\text { dos acumulados, evolução diária das hospitalizações na } \\
\text { rede municipal do Rio de Janeiro. Apresenta as } \\
\text { informações por bairro de origem. }\end{array}$ \\
\hline $\begin{array}{l}\text { CORONA Cidades } \\
\text { (simulacovid.coronacidades.org) }\end{array}$ & USP & $\begin{array}{l}\text { Nacional } \\
\text { (Estados e } \\
\text { Municípios) }\end{array}$ & $\begin{array}{l}\text { Simulador virtual da demanda por hospitais e ventiladores, } \\
\text { do impacto de estratégias de isolamento por cidade e da } \\
\text { capacidade de recursos alocado na intervenção do COVID- } \\
\text { 19. Apresenta dados de casos confirmados, óbitos e } \\
\text { pessoas recuperadas em todas as cidades do país. }\end{array}$ \\
\hline $\begin{array}{l}\text { CORONAVIS } \\
\text { (ufrgs.br/coronavis) }\end{array}$ & UFRGS & \begin{tabular}{|l} 
Nacional \\
(Estados e \\
Municípios)
\end{tabular} & $\begin{array}{l}\text { Painel de controle de casos e mortes confirmados por } \\
\text { dados de vírus corona nos estados e cidades do Brasil. } \\
\text { Apresenta também painel das UTIs em Porto Alegre. }\end{array}$ \\
\hline
\end{tabular}


Tecnologias digitais e de geoprocessamento aplicadas ao monitoramento da doença de Coronovírus 2019 (Covid-19)
Claudio José dos Santos Júnior Paulo José Medeiros de Souza Costa John Victor dos Santos Silva Aryanna Kelly Pinheiro Souza Jackson Pinto Silva

Thiago José Matos Rocha

\begin{tabular}{|c|c|c|c|}
\hline $\begin{array}{l}\text { COVID Goiás } \\
\text { (covidgoias.ufg.br) }\end{array}$ & UFG & Estadual (Goiás) & $\begin{array}{l}\text { Mapa interativo que expressa números de casos } \\
\text { confirmados, suspeitos e óbitos no âmbito dos municípios } \\
\text { do Estado de Goiás-Brasil. Apresentada, também, } \\
\text { incidência de casos por localidade, projeção de casos } \\
\text { novos e de óbitos, informações sobre trafego urbano, } \\
\text { casos confirmados por bairro, mediana do número de } \\
\text { pessoas com doenças respiratórios, além de } \\
\text { geolocalização de unidades de saúde, quantidade de leitos } \\
\text { gerais e de UTI e locais de vacinação contra gripe H1N1. }\end{array}$ \\
\hline $\begin{array}{l}\text { COVID-19 Info Tracking } \\
\text { (camedics.biz/COVID-19-tracking- } \\
\text { info) }\end{array}$ & CAMEDICS & $\begin{array}{l}\text { Nacional } \\
\text { (Estados e } \\
\text { Regiões) }\end{array}$ & $\begin{array}{l}\text { Gráfico interativo atualizado diariamente que expressa } \\
\text { casos confirmados, óbitos, taxa de letalidade, média diária } \\
\text { de casos, variação semanal e aumento \% novos casos por } \\
\text { região do Brasil. }\end{array}$ \\
\hline \begin{tabular}{|l|} 
Epidemia COVID Brasil \\
(guimaraeslabbr.weebly.com/voos)
\end{tabular} & GUIMARAES LAB & $\begin{array}{l}\text { Nacional } \\
\text { (Estados) }\end{array}$ & $\begin{array}{l}\text { Apresenta a vulnerabilidade de cada estado ao contágio } \\
\text { por meio de um índice de centralidade de Katz associado a } \\
\text { rotas diretas e indiretas do território nacional. }\end{array}$ \\
\hline $\begin{array}{l}\text { Pandemia COVID Brasil } \\
\text { (rankbr.com.br/covid19br) }\end{array}$ & RankBR & $\begin{array}{l}\text { Nacional } \\
\text { (Estados e } \\
\text { Municípios) }\end{array}$ & $\begin{array}{l}\text { Apresenta infográficos interativos dos casos no mundo, } \\
\text { avanço de óbitos, casos por estado (infectados, mortes e } \\
\text { letalidade), perfil dos óbitos por grupo de risco, distribuição } \\
\text { dos óbitos por faixa etária e incidência por habitantes para } \\
\text { cada estado do Brasil. }\end{array}$ \\
\hline $\begin{array}{l}\text { Situação COVID-19 Vale do } \\
\text { Ribeira } \\
\text { (localsig.com.br/covid) }\end{array}$ & Local SIG & $\begin{array}{l}\text { Local (Vale do } \\
\text { Ribeira-SP) }\end{array}$ & $\begin{array}{l}\text { Mapa e gráfico interativos apresentam dados de casos } \\
\text { suspeitos, em monitoramento, confirmados, óbitos por } \\
\text { COVID-19 e casos descartados no Sul de São Paulo, na } \\
\text { região do Vale do Ribeira, Brasil. }\end{array}$ \\
\hline $\begin{array}{l}\text { Visão COVID-19 Brasil } \\
\text { (bit.ly/34ol2y3) }\end{array}$ & IBICT & $\begin{array}{l}\text { Nacional } \\
\text { (Estados) }\end{array}$ & $\begin{array}{l}\text { Mapa ilustra total e percentual de casos confirmados, de } \\
\text { casos suspeitos, de óbitos, além de valores diários e } \\
\text { acumulado, por Estado do país, por faixa etária atingida e } \\
\text { por tipo de transmissão, além de informações sobre a rede } \\
\text { hospitalar e de dados de emprego e renda da localidade. }\end{array}$ \\
\hline $\begin{array}{l}\text { Casos e mortes por coronavírus no } \\
\text { Brasil e no Mundo } \\
\text { (bit.ly/34PGu08) }\end{array}$ & G1 (globo.com) & $\begin{array}{l}\text { Nacional } \\
\text { (Estados e } \\
\text { cidades) }\end{array}$ & $\begin{array}{l}\text { Mapa ilustra total de casos e mortes por estado e cidades } \\
\text { do país. Apresenta casos suspeitos por dia, casos } \\
\text { confirmados por dia e cidades com casos. }\end{array}$ \\
\hline
\end{tabular}

Fonte: Elaborado pelos autores (2020).

\section{DISCUSSÃO}

Diante do panorama vivenciado pelo Brasil e pelo mundo de pandemia da COVID-19, consideramos que levantar as ferramentas tecnológicas existentes que viabilizam o acompanhamento e o mapeamento da situação epidemiológica dessa emergente patologia poderia trazer importante contribuição para as ações de vigilância, isolamento, controle e prevenção da doença no âmbito nacional.

Sobre esse tema, importante mencionar que o mapeamento das condições epidemiológicas através do geoprocessamento já vem sendo muito utilizado em outras doenças infecciosas e tem contribuído para as ações de saúde e controle de doenças e agravos à saúde da população brasileira (MARQUES et al., 2017; SANTOS JÚNIOR; SILVA, 2019). 
Tecnologias digitais e de geoprocessamento aplicadas ao monitoramento da doença de Coronovírus 2019 (Covid-19)
Claudio José dos Santos Júnior Paulo José Medeiros de Souza Costa John Victor dos Santos Silva Aryanna Kelly Pinheiro Souza Jackson Pinto Silva

Thiago José Matos Rocha

Tal aspecto torna-se relevante vez que as ferramentas de geoprocessamento são apontadas pela literatura especializada como recursos que facilitam a comunicação, a produção de conhecimento e a tomada de decisão na área de vigilância em saúde (GUIMARÃ̃ES, 2016; CHIARAVALLOTI-NETO, 2016; INHEIRO \& QUEIROZ, 2020; PALMA et al., 2020).

No âmbito do Sistema Único de Saúde (SUS), o mapeamento realizado poderá contribuir para a tomada de decisão no segmento da vigilância de saúde, especificamente no que tange ao direcionamento de recursos, tanto materiais/financeiros quanto de mão de obra profissional, e para acompanhamento dos casos mais graves e de maior necessidade de intervenção no campo da saúde pública (VACONÇELOS; FRIAS, 2017; LANA et al., 2020).

As geotecnologias são aliadas dos sistemas de saúde no mundo e no Brasil. Esses recursos tecnológicos configuram-se como importantes veículos de informações sobre a situação de saúde e epidemiológica, apresentando os índices e incidências de doenças (MARQUES et al., 2017). No âmbito da COVID-19, no Brasil, essas tecnologias podem auxiliar os estados e municípios no mapeamento da situação de regiões, cidades e estados, facilitando as avaliações das medidas tomadas para combater a pandemia até o momento e também as futuras. Esses trabalhos facilitam as ações das secretarias de saúde, principalmente nas medidas de prevenção através do isolamento social (SANTOS JÚNIOR; SILVA, 2019; CABRAL et al., 2020).

O acompanhamento em tempo real da disseminação da pandemia da COVID-19 no mundo tem orientado países nas medidas necessárias para a contenção e prevenção da doença - como é o caso da "quarentena" no Brasil, medida de distanciamento e isolamento social que vem sendo aplicada com intuito de diminuir a incidência do contágio e que começou a ser pensada mediante exemplo de outras ações e de seu sucesso na diminuição da curva de infectados em outros países (VENTURA; AITH; RACHED, 2020).

As evidências geoespaciais e os dados de morbimortalidade acerca da COVID-19 passíveis de serem geradas a partir dos recursos levantados, de forma isolada ou associada a outras técnicas e sistemas de informação geográfica, poderão contribuir para um conjunto de ações de suporte, tomada de decisão, definição de estratégias e prioridades por parte dos setores de vigilância epidemiológica (CABRAL et al., 2020; RIBEIRO et al., 2014).

As tecnologias de geoprocessamento apresentadas neste trabalho poderão ser utilizadas com fim de orientar as seguintes ações em saúde no âmbito nacional: análise da distribuição espacial do agravo em regiões de interesse em saúde pública; avaliação da abrangência, influências externas e da territorialidade dos casos e óbitos; avaliação de associação da patologia com indicadores habitacionais e socioeconômicos; análise do avanço territorial e espaço-temporal dos casos e óbitos; análise de padrões, aglomerados e áreas de risco; avaliação da dinâmica de propagação; análise da demanda de pacientes e da disponibilidade de serviços de saúde; avaliação da relação de adoecimentos com variáveis ambientais e de infraestrutura; análise de dependência espacial; previsão de cenários e da capacidade de recursos humanos e materiais, entre outras aplicações.

A incerteza e o desconhecimento, importantes características que marcam atualmente o comportamento da pandemia de COVID-19, são fatores que podem ser minimizados mediante a adoção de ações planejadas de vigilância em saúde. Espera-se, assim, que as tecnologias aqui apresentadas, complementadas por outros estudos observacionais, clínicos, randomizados e de revisão sistemática, possam servir de suporte para elucidar o panorama de dúvidas que predomina em relação ao comportamento do novo coronavírus.

\section{CONCLUSÃO}

O estudo consistiu num levantamento desenvolvido em meio à pandemia mundial da infecção por SARS-CoV-2, reunindo e indicando um rico arsenal com as principais tecnologias de mapeamento, monitoramento e disseminação de informações geográfico-epidemiológicas sobre a COVID-19, tendo sido realizado por meio da pesquisa bibliométrica.

As ferramentas de tecnologias de geoprocessamento aqui levantadas podem ser empregadas como recurso e/ou meio de divulgação de informações epidemiológicas atualizadas sobre a pandemia da 
Tecnologias digitais e de geoprocessamento aplicadas ao monitoramento da doença de Coronovírus 2019 (Covid-19)
Claudio José dos Santos Júnior Paulo José Medeiros de Souza Costa John Victor dos Santos Silva Aryanna Kelly Pinheiro Souza Jackson Pinto Silva

Thiago José Matos Rocha

COVID-19 para a comunidade em geral e indivíduos interessado no tema, como instrumento de consulta às fontes de informação geoespaciais acerca de dados morbimortalidade da pandemia por SARS-CoV-2 e também como ferramenta auxiliar e de suporte às atividades de vigilância em saúde pelos setores das secretarias e departamentos de saúde de diferentes âmbitos.

Estudos de avaliação dessas ferramentas tecnológicas e do impacto das mesmas na vigilância em saúde no Brasil e na contenção da COVID-19 são possibilidades de novos trabalhos neste segmento.

\section{REFERÊNCIAS}

ARREAZA, A. L. V.; MORAES, J. C. Vigilância da saúde: fundamentos, interfaces e tendências. Ciência e Saúde Coletiva, v.15, n. 4, p. 2215-2228, 2010. https://doi.org/10.1590/S141381232010000400036

BONIFÁCIO, S. R.; LOPES, E. L. Mapping of health problems: an application of the georeferencing technique using the Google Earth software. International Journal of Health Management, v. 2, n. 1, p. 1-16, 2019.

BRASIL. Lei $n^{\circ} 8.080$, de 19 de setembro de 1990. Dispõe sobre as condições para a promoção, proteção e recuperação da saúde, a organização e o funcionamento dos serviços correspondentes e dá outras providências. Diário Oficial da União, Brasília, 19 set. 1990.

CABRAL, R. B. G. et al. Estudo inicial sobre a evolução do novo CORONAVÍRUS (SARS-COV-2) no estado do Pará (Brasil), no período entre 17/03/2020 e 06/04/2020. Brazilian Journal of Health

Review, v. 3, n. 2, p. 2914-2931, 2020. https://doi.org/10.34119/bjhrv3n2-133

CARVALHO, I. S. A participação do homem durante o nascimento do filho: vídeos do Youtube. Revista Enfermagem UERJ, v. 25, p. 1-6, 2017. https://doi.org/10.12957/reuerj.2017.11474

CHIARAVALLOTI-NETO, F. O geoprocessamento e saúde pública. Arquivos de Ciências da Saúde, v. 23, n. 4, p. 1-2, 2016. https://doi.org/10.17696/2318-3691.23.4.2016.661

GOLDSTEIN, R. A. et al. A experiência de mapeamento participativo para a construção de uma alternativa cartográfica para a ESF. Ciência e Saúde Coletiva, v. 18, n. 1, p. 45-56, 2013. ttps://doi.org/10.1590/S1413-81232013000100006

GUIMARÃES, R. B. Geografia e Saúde Coletiva no Brasil. Saúde e Sociedade, v. 25, n. 4, p. 869879, 2016. https://doi.org/10.1590/s0104-12902016167769

LANA, R. M. et al. Emergência do novo coronavírus (SARS-CoV-2) e o papel de uma vigilância nacional em saúde oportuna e efetiva. Cadernos de Saúde Pública, v. 36, p. e00019620, 2020. https://doi.org/10.1590/0102-311x00019620

MARCELO, J. F.; HAYASHI, M. C. P. I. Estudo bibliométrico sobre a produção científica da área da sociologia da ciência; Estudio bibliométrico en la producción científica del campo de la sociología de la ciencia. Informação e Informação, v. 18, n. 3, p. 138-153, 2013. https://doi.org/10.5433/1981$\underline{8920.2013 v 18 n 3 p 138}$

MURUGIAH, K. et al. YouTube as a source of information on cardiopulmonary resuscitation.

Resuscitation, v. 82, n. 3, p. 332-4, 2011. https://doi.org/10.1016/j.resuscitation.2010.11.015

PALMA, D. C. A.; SANTOS, E. S.; IGNOTTI, E. Análise dos padrões espaciais e caracterização dos suicídios no Brasil entre 1990 e 2015. Cadernos de Saúde Pública, v. 36, n. 4, p. 1-13, 2020. https://doi.org/10.1590/0102-311x00092819

PINHEIRO, P. C.; QUEIROZ, B. L. Análise espacial da mortalidade por acidentes de motocicleta nos municípios do Brasil. Ciência e Saúde Coletiva, v. 25, n. 2, p. 683-692, 2020.

https://doi.org/10.1590/1413-81232020252.14472018

RIBEIRO, M. A. et al. Geoprocessamento em saúde como tecnologia de análise e monitoramento da hanseníase no município de Sobral-Ceará. Revista Baiana de Saúde Pública, v. 41, n. 2, p. 451465, 2018. https://doi.org/10.22278/2318-2660.2017.v41.n2.a2506 
RIBEIRO, M. A. et al. Georreferenciamento: ferramenta de análise do sistema de saúde de SobralCeará. SANARE-Revista de Políticas Públicas, v. 1, n. 2, p. 63-69, 2014.

SANTOS JÚNIOR, C. J.; SILVA, J. P. Epidemiologia, fatores climáticos e distribuição espacial da Dengue em uma capital do Nordeste do Brasil. Revista Brasileira de Climatologia, v. 25, p. 1-14, 2019. https://doi.org/10.5380/abclima.v25i0.69421

SILVA, J. B.; MUNIZ, A. M. V. Pandemia do Coronavírus no Brasil: Impactos no Território Cearense. Espaço e Economia. Revista Brasileira de Geografia Econômica, v. 9, 17, 2020. https://doi.org/10.4000/espacoeconomia.10501

VASCONCELOS, C. S.; FRIAS, P. G. Avaliação da Vigilância da Síndrome Gripal: estudo de casos em unidade sentinela. Saúde em Debate, v. 41, p. 259-274, 2017. https://doi.org/10.1590/0103$11042017 \mathrm{~s} 19$

VENTURA, D. F. L.; AITH, F. M. A.; RACHED, H. A emergência do novo coronavírus e a "lei de quarentena" no Brasil. Revista Direito e Práxis, Ahead of print, 2020.

WORLD HEALTH ORGANIZATION (WHO). Coronavirus disease (COVID-19) situation. Disponível em: www.who.int/emergencies/diseases/novel-coronavirus-2019. Acesso em 17 mai. 2020. 